\title{
Identification of Prognostic Factors and Risk Groups in Patients Found to Have Nodal Metastasis at the Time of Radical Hysterectomy for Early- Stage Squamous Carcinoma of the Cervix ${ }^{1}$
}

\author{
Ronald D. Alvarez, M.D., ${ }^{*}$ Seng-Jaw Soong, Ph.D., ${ }^{*}$ Walter K. Kinney, M.D. $\dagger$ Gary C. Reid, M.D. $\ddagger$ Mark \\ F. Schray, M.D. $\dagger$ K Karl C. Podratz, M.D., Ph.D. $\dagger$ George W. Morley, M.D. $\neq$ \\ and Hugh M. Shingleton, M.D.* \\ *University of Alabama, at Birmingham, Birmingham, Alabama 35294; $\uparrow$ Mayo Clinic, Rochester, Minnesota 55905; and \\ $\ddagger$ University of Michigan, Ann Arbor, Michigan 48109
}

Received May 12, 1989

\begin{abstract}
In a retrospective study conducted at the University of Alabama at Birmingham, the University of Michigan, and the Mayo Clinic, 185 patients with previously untreated FIGO stage IB and IIA squamous cell carcinoma of the cervix were found to have nodal metastasis at the time of radical hysterectomy and pelvic lymphadenectomy. Of these patients, 103 received adjuvant pelvic irradiation. Cancer recurred in 76 patients; the median time to recurrence was 3.1 years. The prognostic significance of patient age, clinical stage, lesion diameter, number and location of nodal metastases, and use of adjuvant radiation therapy was determined by multivariate analysis. Only patient age $(P=0.0006)$, lesion diameter $(P<0.0001)$, and number of nodal metastases $(P=$ 0.0004 ) were noted to be significant factors in determining overall survival. Rates of recurrence were also related to these factors. Employment of these significant variables led to identification of four risk groups. In general, patients with small cervical lesions (diameter $<1 \mathrm{~cm}$ ) and no more than two nodes with metastases fell into the low-risk category; those patients with large cervical lesions (diameter $>4 \mathrm{~cm}$ ) and more than two involved nodes fell into the high-risk category. All other patients were categorized into intermediate-risk groups. Ten-year survival was $92 \%$ in the low-risk group $(n=13), 70 \%$ in the low-intermediate-risk group $(n=66), 56 \%$ in the high-intermediate-risk group $(n=66)$, and $13 \%$ in the high-risk group $(n=20)$. This risk group classification identifies subgroups of early-stage cervical carcinoma patients found to have nodal metastasis at the time of radical hysterectomy that warrant appropriately selected adjuvant therapy. 1989 Academic Press, Inc.
\end{abstract}

\section{INTRODUCTION}

Of all histopathologic features, the presence of lymph node metastasis has been described as perhaps the most

\footnotetext{
${ }^{1}$ Presented at the 1989 Annual Meeting of the American College of Obstetrics and Gynecology.
}

significant prognostic factor in those patients undergoing radical hysterectomy for early-stage cervical carcinoma $[1$, p. $148 ; 2,3]$. Although adjuvant pelvic irradiation is employed by many clinicians in this particular situation, controversy exists over the value of such treatment in terms of overall survival [4-10]. Systemic therapy has been advocated by some $[1$, p. $148 ; 11,12]$, yet no prospective randomized studies have been reported. Longterm survival occurs in many patients even in the absence of adjuvant treatment [13]. The purpose of this report is to identify the patients with early-stage squamous cell carcinoma of the cervix found to have pelvic node metastasis at the time of radical hysterectomy and lymphadenectomy who are at significant risk for tumor recurrence and are therefore potential candidates for adjuvant therapy.

\section{MATERIALS AND METHODS}

A retrospective review of all patients with early-stage squamous carcinoma of the cervix who were treated by radical hysterectomy and pelvic lymphadenectomy was conducted at the University of Alabama at Birmingham (UAB), the University of Michigan (UM), and the Mayo Clinic (MC). Nodal metastasis was identified in 185 patients -48 from UAB, 42 from UM, and 95 from MC. The medical records of these patients were reviewed with special emphasis on the clinicopathologic findings at the time of initial presentation and treatment and, when applicable, at the time of tumor recurrence. Current followup status was obtained by review of the patient record, by written or telephone correspondence, or by review of the death certificate. 
The median age of the 185 patients found to have nodal metastasis at the time of radical hysterectomy was 42 years. FIGO stage IB was documented in 150 patients; the remaining 35 patients were assigned FIGO stage IIA. Median tumor diameter was $3.0 \mathrm{~cm}$, with a range of 0.5 to $9.5 \mathrm{~cm}$. The total number of metastatically involved nodes ranged from 1 to 35 : 95 patients had metastasis to a single node and only one patient had more than 12 involved nodes. Bilateral pelvic nodal involvement was observed in 47 patients. Paraaortic nodes were sampled in 137 patients; only 5 patients were found to have metastasis to the paraaortic nodal region.

Postoperative adjuvant pelvic irradiation was administered to 103 of 185 patients ( $56 \%$ ). The median midplane dose of pelvic irradiation was $5000 \mathrm{cGy}$, with $75 \%$ of patients treated with adjuvant pelvic irradiation receiving greater than or equal to $5000 \mathrm{cGy}$. Median follow-up time was 4.7 years. Recurrence was documented in 76 patients with a median disease-free interval of 3.1 years. Isolated pelvic failures occurred in 27 patients; 37 patients had isolated distal recurrence. Six patients had both pelvic and distal recurrence; the site of recurrence was not specified in 6 of the 76 patients with recurrence. Of the 76 patients who had recurrence, 43 had received adjuvant pelvic irradiation postoperatively.

The prognostic significance of patient age, clinical stage, tumor diameter, number and location of nodal metastases, and use of adjuvant radiation therapy was studied by univariate and multivariate analysis and expressed in terms of both survival and recurrence. The survival curves were calculated using the method of Kaplan and Meier, and the log-rank test was employed to determine the level of significance. With postoperative adjuvant treatment employed as the stratification variable, the multivariate analysis was performed using the rank regression procedure described by Kalbfleisch and Prentice [14]. $\chi^{2}$ analysis was also utilized to compare recurrence and survival rates. A result was deemed significant when $P$ was less than 0.05 .

\section{RESULTS}

The results of our analysis demonstrated no significant institutional differences in overall survival. When analyzed separately as a single factor, patient age $(P<$ $0.0001)$ and tumor diameter $(P<0.0001)$ were found to significantly correlate with patient survival (Figs. 1 and 2). Clinical stage was also noted to significantly correlate with patient survival: 5-year survival was $75 \%$ in FIGO stage IB patients and $62 \%$ in FIGO stage IIA patients $(P=0.0096)$. Although there was no significant association of survival with the number of nodal metastases, there was a trend toward poorer survival in those patients with involvement of more than two lymph nodes. There

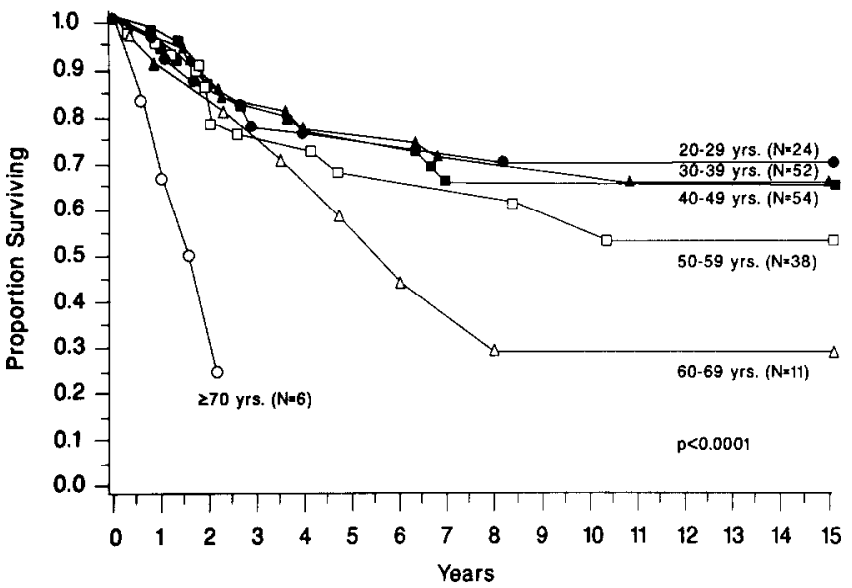

FIG. 1. Early-stage cancer of the cervix with nodal metastasis survival according to age group.

was no significant association of survival with unilateral versus bilateral nodal metastasis. Four of five patients with metastasis to periaortic nodes died, three of disease and one of complications related to adjuvant radiotherapy. The significance of metastasis to nodes above the level of the common iliac vessels was difficult to delineate in this study due to the small number of patients with metastasis to that region. There was no survival benefit to adjuvant radiation therapy: the significance of this finding has been delineated in a matched, controlled study described elsewhere [4].

In the multivariate analysis, when all factors were simultaneously assessed, the number of nodal metastases $(P=0.0004)$ proved to be a significant variable in determining survival, in addition to patient age $(P=0.006)$ and tumor diameter $(P<0.0001)$ (Table 1). Clinical stage was not a significant factor in determining survival after adjustment for other factors. Similar clinico-pathologic factors were determined to be significant with respect to

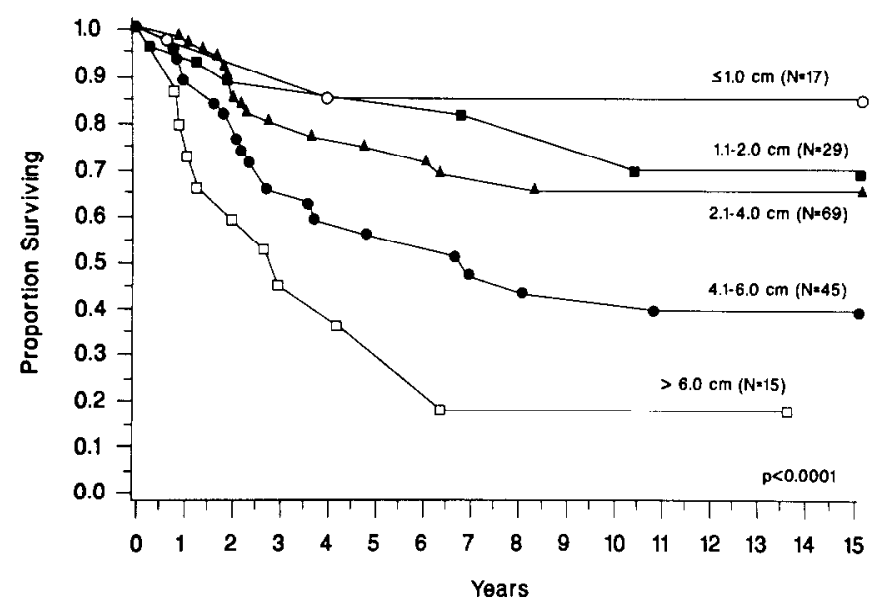

FIG. 2. Early-stage cancer of the cervix with nodal metastasis: survival according to tumor diameter. 
TABLE 1

Prognostic Factor Analysis

\begin{tabular}{|c|c|c|c|c|}
\hline \multirow[b]{2}{*}{ Factor } & \multicolumn{2}{|c|}{$\begin{array}{c}\text { Univariate } \\
\text { analysis }\end{array}$} & \multicolumn{2}{|c|}{$\begin{array}{l}\text { Multivariate } \\
\text { analysis }{ }^{\prime \prime}\end{array}$} \\
\hline & $x^{2}$ & $\boldsymbol{P}$ & $x^{2}$ & $P$ \\
\hline Age & 15.7 & $<0.0001$ & 11.8 & 0.006 \\
\hline Tumor diameter & 22.0 & $<0.0001$ & 22,0 & $<0.0001$ \\
\hline Clinical stage & 6.7 & 0.0096 & 0.2 & 0.6790 \\
\hline $\begin{array}{l}\text { Number of nodes with } \\
\text { metastasis }\end{array}$ & 2.5 & 0.1139 & 12.4 & 0.0004 \\
\hline $\begin{array}{l}\text { Bilateral vs } \\
\text { unilateral nodes } \\
\text { with metastasis }\end{array}$ & 1.1 & 0.2973 & 2.2 & 0.1355 \\
\hline
\end{tabular}

${ }^{a}$ Based on the rank regression analysis with stratification for treatment (surgery only vs surgery and radiation).

recurrence. In univariate analysis, patient age $(P-$ $0.0049)$, tumor diameter $(P<0.0001)$, and number of nodal metastases $(P=0.0023)$ were significant factors in determining the rate of recurrence; these factors remained significant after adjustment for other variables (Table 2).

By employment of these independently significant clinical variables, risk groups could be formulated. Patients could be categorized into groups of low, low-intermediate, high-intermediate, and high risk. The characteristics of each group are demonstrated in Table 3. The number of patients in each group receiving adjuvant radiotherapy is given in Table 4. Ten-year survival was $92 \%$ in the low-risk group $(n=13), 70 \%$ in the lowintermediate-risk group $(n=66), 56 \%$ in the high-intermediate-risk group $(n=66)$, and $13 \%$ in the high-risk group $(n=20)$ (Fig. 3$)$. There was a significant difference in survival rates between the low-risk and intermediaterisk groups, the intermediate-risk groups and the high-

TABLE 2

Recurrence Rates According to Prognostic Clinical Variables

\begin{tabular}{lccc}
\hline Clinical variables & Number of patients & Recurrence rate $(\%)$ & $P^{u}$ \\
\hline Age (years) & & & \\
$20-49$ & 130 & 35 & \\
$50-69$ & 49 & 53 & 0.008 \\
$\geqslant 70$ & 6 & 83 & \\
Tumor diameter (cm) & & & \\
$\leqslant 1.0$ & 17 & 18 & \\
$1.1-2.0$ & 29 & 31 & \\
$2.1-4.0$ & 69 & 38 & \\
$4.1-6.0$ & 45 & 58 & \\
$>6.0$ & 15 & 67 & \\
Number of nodes with metastasis & & \\
1 & 95 & 32 & \\
2 & 33 & 42 & \\
$>2$ & 47 & 62 & 0.006 \\
\hline
\end{tabular}

${ }^{a}$ Determined by multivariate analysis.
TABLE 3

Definition of Risk Groups ${ }^{a}$

\begin{tabular}{lccc}
\hline \multirow{2}{*}{$\begin{array}{l}\text { Number of nodes } \\
\text { with metastasis }\end{array}$} & $<1 \mathrm{~cm}$ & $1.1-4 \mathrm{~cm}$ & $>4 \mathrm{~cm}$ \\
\cline { 2 - 4 } & LR & IR1 & IR2 \\
$>2$ & IR1 & IR2 & HR \\
\hline
\end{tabular}

${ }^{"}$ LR, low risk; IR1, low intermediate risk; IR2, high intermediate risk; HR, high risk.

${ }^{b}$ This group includes only patients under age 60 . All patients 60 years and older with cervical lesions larger than $4 \mathrm{~cm}$ in diameter and with two or fewer involved nodes were classified in the high-risk group.

risk group, and, obviously, the low-risk and high-risk groups $(P<0.0001)$. Recurrence rates were $8 \%$ in the low-risk group, $29 \%$ in the low-intermediate-risk group, $53 \%$ in the high-intermediate-risk group, and $80 \%$ in the high-risk group $(P<0.0001)$.

\section{DISCUSSION}

Carcinoma metastatic to regional lymph nodes is a poor prognostic indicator in patients undergoing radical hysterectomy for early-stage squamous cell carcinoma of the cervix. Overall survival in this group of patients is stated to be 50 to $60 \%[2,3,15]$. In an effort to delineate risk groups in patients with cervical carcinoma found to have pelvic node metastases, the prognostic significance of several nodal characteristics has been investigated. A summary of several reports regarding the prognostic significance of number of nodal metastases, metastasis to nodes in the region of the common iliac vessels and above, and bilateral pelvic nodal metastasis as opposed to unilateral involvement is included in Table 5. Most of these reports conclude that extensive nodal metastasis or metastasis to the nodes in the region of the common iliac vessels and above are poor prognostic nodal characteristics. Whether bilateral nodal metastasis connotes a less favorable prognosis relative to unilateral involvement remains unresolved. Other nodal characteristics that have been suggested as prognostic indicators include dimensions of the metastatically involved nodes and resectability of the involved nodes $[22,23]$.

TABLE 4

Distribution of Patients in Each Risk Group Receiving Adjuvant Radiotherapy

\begin{tabular}{lrr}
\hline & \multicolumn{3}{c}{$\begin{array}{c}\text { Postoperative } \\
\text { radiotherapy }\end{array}$} \\
\cline { 2 - 4 } Risk group & No & Yes \\
\hline Low & 5 & 8 \\
Low-intermediate & 29 & 37 \\
High-intermediate & 33 & 33 \\
High & 3 & 17 \\
\hline
\end{tabular}




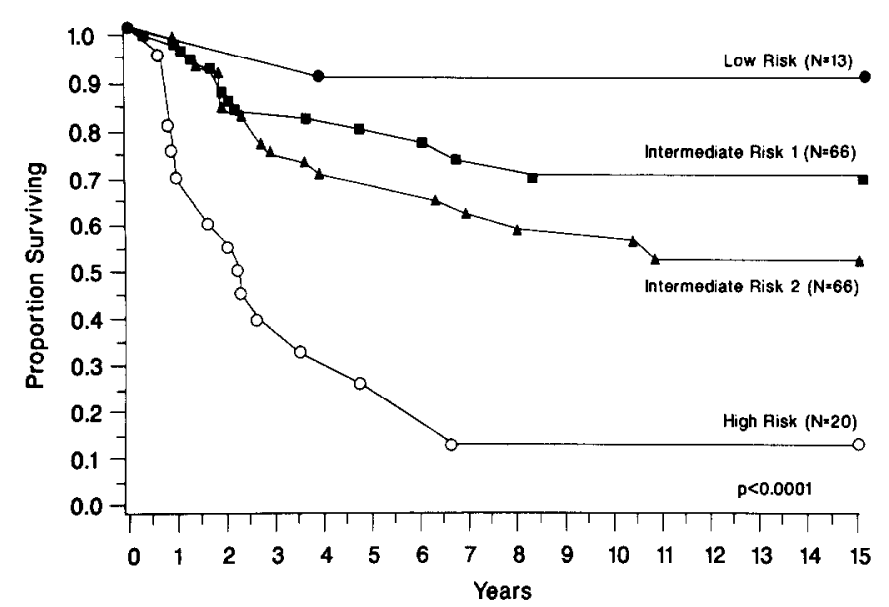

FIG. 3. Early-stage cancer of the ccrvix with nodal metastasis: survival according to risk group.

Too little attention has been focused on the prognostic significance of characteristics of the primary cervical lesion in patients with early-stage cervical cancer found to have nodal metastasis at the time of radical hysterectomy. Many of these characteristics, i.e., lesion diameter, depth of invasion, lymph-vascular space invasion, and parametrial extension, are known to be associated with a high risk of nodal metastasis and with a high rate of tumor recurrence even in the absence of nodal metastasis $[12,19,24-26]$. In a study of 71 earlystage cervical carcinoma patients found to have nodal metastasis at the time of radical hysterectomy, Fuller $e t$ al. [21] noted clinical stage, cervical lesion diameter, tumor histology, and parametrial or uterine extension to be prognostically significant tumor characteristics. Specifically, 20 of 44 patients (45\%) with cervical carcinoma lesions $3 \mathrm{~cm}$ or larger recurred as opposed to 9 of 26 patients (35\%) with lesions smaller than $3 \mathrm{~cm}$; however, no statistical analysis was provided. In a study of 25 patients with nodal metastases, Chung et al. [12] noted an extremely high rate of both local and distant recurrences in those patients with bulky primary tumors, but no statistical correlation according to cervical lesion diameter in patients with nodal metastasis was provided.

TABLE 5

Prognostic Characteristics of Metastatically Involved Nodes: Review of the Literature

\begin{tabular}{|c|c|c|c|c|c|}
\hline \multirow[b]{2}{*}{ Author } & \multirow[b]{2}{*}{$\begin{array}{l}\text { Clinical } \\
\text { stage }\end{array}$} & \multirow[b]{2}{*}{$\begin{array}{l}\text { Number } \\
\text { of } \\
\text { patients }\end{array}$} & \multicolumn{3}{|c|}{$\begin{array}{l}\text { Prognostic characteristics } \\
\text { of nodes with metastasis" }\end{array}$} \\
\hline & & & $\begin{array}{l}\text { Number of } \\
\text { nodes }\end{array}$ & $\begin{array}{l}\text { Location } \\
\text { of nodes }\end{array}$ & $\begin{array}{c}\text { Bilateral vs } \\
\text { unilateral } \\
\text { involvement }\end{array}$ \\
\hline Pilleron et al. [15] & I & 68 & $\mathbf{S}^{h}$ & $\mathrm{~S}$ & $\mathrm{~S}$ \\
\hline Hsu et al. [16] & I & $\begin{array}{l}37 \\
50\end{array}$ & $S$ & $S$ & $\mathrm{~S}$ \\
\hline Martimbeau et al. [17] & IB & 120 & NS & $S$ & NS \\
\hline Tanaka et al. [18] & $\begin{array}{l}\text { IB } \\
\text { II }\end{array}$ & $\begin{array}{r}5 \\
46\end{array}$ & $\mathrm{~S}$ & $\mathrm{~S}$ & $\mathrm{~S}$ \\
\hline Morrow [5] & IB & 195 & $\mathrm{~S}$ & NA & NA \\
\hline Webb and Symmonds [2] & $\begin{array}{l}\text { IB } \\
\text { IIA }\end{array}$ & $\begin{array}{l}14 \\
27\end{array}$ & NA & NA & NS \\
\hline Kim et al. [20] & IB & 23 & $S$ & $\mathrm{NA}$ & NA \\
\hline Fuller et al. [21] & $\begin{array}{l}\text { IB } \\
\text { IIA }\end{array}$ & $\begin{array}{l}42 \\
29\end{array}$ & $\mathrm{~S}$ & NA & NA \\
\hline
\end{tabular}

${ }^{a}$ As determined by univariate analysis.

${ }^{b} \mathrm{~S}$, significant; NS, not significant; NA, not analyzed. 
No other report to date has investigated the prognostic significance of the aforementioned characteristics of the primary cervical lesion in patients found to have nodal metastasis at the time of radical hysterectomy.

In this study, we have investigated the prognostic significance of characteristics of both the nodal metastases and the primary cervical lesion. The number of metastatically involved nodes and the diameter of the primary lesion were the histopathologic features that readily identified prognostically significant risk groups. These two factors reflect the impact of both the primary lesion and the secondary site tumor burden in determining prognosis. Our data would suggest that the patients with small tumor dimensions (diameter $<1.0 \mathrm{~cm}$ ) and with one to two involved nodes have an excellent prognosis and are adequately treated with radical hysterectomy and pelvic lymphadenectomy. Adjuvant pelvic irradiation is probably unnecessary in this group. Conversely, patients with large tumor dimensions (diameter $>4.0 \mathrm{~cm}$ ) and more than two involved nodes are at high risk for tumor recurrence and have compromised survival. This high-risk group, therefore, warrants aggressive adjuvant treatment with systemic therapy in the presence or absence of pelvic radiotherapy. For those patients designated as being at intermediate risk, adjuvant pelvic irradiation may be beneficial in affording local pelvic tumor control but, as previously stated, the value of such adjuvant treatment in terms of overall survival remains unresolved [4-10].

Confirming the observations of Fuller et al. [21], the present study demonstrated an inverse correlation between survival and age in patients found to have lymph node metastasis at the time of radical hysterectomy. The significance of age in patients with cervical carcinoma has been extensively investigated but, nevertheless, remains unresolved [1, p. 123]. Our results might suggest that the elderly patient with large tumor dimensions and multiple nodal metastases is either more immunologically compromised or less able to tolerate aggressive adjuvant or salvage therapy than younger patients.

In conclusion, this report is the initial assessment employing a multivariate analysis of the prognostic significance of the characteristics of both the primary cervical lesion and the regional lymph nodes in patients with early-stage squamous cervical carcinoma found to have nodal metastasis at the time of radical hysterectomy. These analyses have facilitated the identification of specific groups of patients at high risk for tumor recurrence and poor overall survival who warrant innovative adjuvant therapy. Further investigation of the significance of other clinical histopathologic risk factors in discriminating between risk groups and future controlled prospective studies of adjuvant therapy in both intermediateand high-risk groups are warranted.

\section{REFERENCES}

1. Shingleton, H. M., and Orr, J. W. (Eds.) Cancer of the cervixDiagnosis and treatment, Churchill Livingstone, New York (1987).

2. Webb, M. J., and Symmonds, R. E. Wertheim hysterectomy: A reappraisal, Obstet. Gynecol. 54, 140-145 (1979).

3. Masubuchi, K., Tenjin, Y., Kubo, H., and Kimura, M. Five-year cure rate for carcinoma of the cervix uteri, Amer. J. Obstet. Gynecol. 103, 566-573 (1969).

4. Kinney, W. K., Alvarez, R. D., Reid, G. C., Schray, M. F., Soong, S-J., Morley, G. W., Podratz, K. C., and Shingleton, H. M. Value of adjuvant whole pelvic irradiation for early stage squamous carcinoma of the cervix with pelvic nodal metastasis: A matched controlled study, Gynecol. Oncol (1989).

5. Morrow, C. P. (moderator). Panel report: Is pelvic radiation beneficial in the postoperative management of Stage IB squamous cell carcinoma of the cervix with pelvic node metastasis treated by radical hysterectomy and pelvic lymphadenectomy? Gynecol. Oncol. 10, 105-110 (1980).

6. Hogan, W. M., Littman, P., Grincr, L., Miller, C. L., and Mikuta, J. J. Results of radiation therapy given after radical hysterectomy, Cancer 49, 1278-1285 (1982).

7. Larson, D. M., Stringer, C. A., Copeland, L. J., Gershenson D. M., Malone, J. M., and Rutledge, F. N. Stage IB cervical carcinoma treated with radical hysterectomy and pelvic lymphadenectomy: Role of adjuvant radiotherapy, Obstet. Gynecol. 69, 378381 (1987).

8. Guttman, R. Significance of postoperative irradiation in carcinoma of the cervix: A ten year survey, Amer. J. Roentgenol. 108, 102108 (1970).

9. Himmelmann, A., Holmberg. E., Jansson, I., Oden, A., and Skogsberg, $K$. The effect of postoperative external radiotherapy on cervical carcinoma stage IB and IIA, Gynecol. Oncol. 22, 73-84 (1985).

10. Russell, A. H., Tong, D. Y., Figge, D. C., Tamimi, H. K., Greer, B. E., and Elder, S. J. Adjuvant postoperative pelvic radiation for carcinoma of the uterine cervix: Pattern of cancer recurrence in patients undergoing elective radiation following radical hysterectomy and pelvic lymphadenectomy, Int. J. Radiat. Oncol. Biol. Phys. 10, 211-214 (1984).

11. Barter, J. F., Soong, S-J., Shingleton, H. M., Hatch, K. D., and Orr, J. W. Complications of combined radical hysterectomy-postoperative radiation therapy in women with early stage cervical cancer, Gynecol. Oncol. 32, 292-296 (1989).

12. Chung, C. K., Nahhas, W. A., Stryker, J. A., Curry, S. L., Abt, A. B., and Mortcl, R. Analysis of factors contributing to treatment failures in stages IB and IIA carcinoma of the cervix, Amer. J. Obstet. Gynecol. 138, 550-556 (1980).

13. Delgado, G. Stage IB squamous cancer of the cervix: The choice of treatment, Obstet. Gynecol. Surv. 33, 174-183 (1978).

14. Kalbfleisch, J. D., and Prentice, P. L. The statistical analysis of failure time data, Wiley, New York (1983).

15. Pilleron, J. P., Durand, J. C., and Hamelin, J. P. Prognostic value of node metastasis in cancer of the uterine cervix, Amer. J. Obstet. Gynecol. 119, 458-462 (1974).

16. Hsu, C-T., Cheng, Y-S., and Su, S-C. Prognosis of uterine cervical cancer with extensive lymph node metastases, Amer. I. Obstet. Gynecol. 114, 954-962 (1972).

17. Martimbeau, P. W., Kjorstad, K. E., and Iversen, T. Stage IB carcinoma of the cervix, the Norwegian Radium Hospital. II. Results when pelvic nodes are involved, Obstet. Gynecol. 60, 215218 (1982). 
18. Tanaka, Y., Sawada, S., and Murata, T. Relationship between lymph node metastases and prognosis in patients irradiated postoperatively for carcinoma of the uterine cervix, Acta Radiol. Oncol. 23, 455-459 (1984).

19. Piver, M. S., and Chung, W. S. Prognostic significance of cervical lesion size and pelvic node metastases in cervical carcinnma, $O b$ stet. Gynecol. 46, 507-510 (1975).

20. Kim, R. Y., Salter, M. M., and Shingleton, H. M. Adjuvant postoperative radiation therapy following radical hysterectomy in stage IB cancer of the cervix-Analysis of treatment failure, Int. J. Radiat. Oncol. 14, 445-449 (1988).

21. Fuller, A. F., Elliott, N., Kosloff, C., and Lewis, J. L. Lymph node metastases from carcinoma of the cervix, stages IB and IIA: Implications for prognosis and treatment, Gynecol. Oncol. 13, 165174 (1982).

22. Inoue, T., Chihara, T., and Morita, $K$. The prognostic significance of the size of the largest nodes in metastatic carcinoma from the uterine cervix, Gynecol. Oncol. 19, 187-193 (1984).

23. Downey, G. O., Potish, R. A., Adcock, L. L., Prem, K. A., and Twiggs, L. B. Pretreatment surgical staging in cervical carcinoma: Therapeutic efficacy of pelvic lymph node dissection, Gynecol. Oncol. (1989).

24. Shingleton, H. M., Gore, H., Soong, S-J., Orr, J. W., Jr., Hatch, K. D., Austin, J. M., Jr., and Patridge, E. E. Tumor recurrence and survival in stage Ib cancer of the cervix, Amer. J. Clin. Oncol. 6, 265-272 (1983).

25. Inoue, T., and Okumura, M. Prognostic significance of parametrial extension in patients with cervical carcinoma stages IB, IIA, and IIB. Cancer 54, 1714-1719 (1984).

26. Delgado, G., Bandy, B. N., Fowler, W. C., Stehman, F. B., Sevin, B., Creasman, W. T., Major, F., DiSaia, R., and Zaino, R. A prospective surgical pathological study of stage I squamous carcinoma of the cervix, Gynecol. Oncol. (1989). 